\title{
Circuit
}

Musiques contemporaines

\section{Petite apologie de l'art des sons fixés}

\section{A brief apologia of the art of fixed sounds}

\section{Francis Dhomont}

Volume 4, numéro 1-2, 1993

Électroacoustique-Québec : l'essor

URI : https://id.erudit.org/iderudit/902066ar

DOI : https://doi.org/10.7202/902066ar

Aller au sommaire du numéro

Éditeur(s)

Les Presses de l'Université de Montréal

ISSN

1183-1693 (imprimé)

1488-9692 (numérique)

Découvrir la revue

Citer cet article

Dhomont, F. (1993). Petite apologie de l'art des sons fixés. Circuit, 4(1-2), 55-66. https://doi.org/10.7202/902066ar

\section{Résumé de l'article}

Défense et illustration, par le responsable du présent numéro, de l'option acousmatique face aux critiques et aux procès dont elle fait l'objet. d'utilisation que vous pouvez consulter en ligne.

https://apropos.erudit.org/fr/usagers/politique-dutilisation/ 


\title{
Petite apologie de l'art des sons fixés ${ }^{(1)}$
}

\author{
Francis Dhomont
}

Actuellement la musique classique constitue un goût spécial, comme la langue grecque ou l'archéologie précolombienne, et non pas une culture commune, un fonds instinctif de communication réciproque et une sténographie psychologique.

(Allan Bloom, L'âme désarmée, Essai sur le déclin de la culture générale.)

\section{Illustration...}

Bien que François Guérin ait déjà clairement esquissé ici les contours de l'acousmatique(2), peut-être convient-il néanmoins $d^{\prime}$ insister un peu sur la signification du vocable lui-même et sur ses acceptions successives. Que le lecteur qui n'ignore plus rien de ces notions veuille bien m'excuser et sauter, s'il le souhaite, ces quelques prolégomènes.

L'origine du mot est fort ancienne mais, au sens moderne, il désigne un genre électroacoustique répondant à un ensemble de gestes compositionnels spécifiques, à des conditions d'écoute/réalisation et à des stratégies de projection dans l'espace qui lui sont propres. Tentant une synthèse de divers points de vue (ce qui s'avère plus complexe qu'il n'y paraît), voici ce que j'écrivais dans un texte récent:

Ce terme est attribué à Pythagore $\mid V^{e}{ }^{e}$ siècle avant J.-C.) qui dispensait, diton, son enseignement - uniquement oral - dissimulé derrière une tenture afin que ses disciples ne soient pas distraits par sa présence
(1) Titre inspiré de celui de Michel Chion: L'Art des sons fixés ou la Musique concrètement, 1991, Fontaine, Éditions Métamkine/ Nota-Bene/Sono-Concept.
(2) Voir ci-dessus l'article de François Guérin «Aperçu du genre électroacoustique au Québec», pp. 9-31. La définition qui figure le plus couramment dans certains dictionnaires dit à peu près ceci : "Son que l'on perçoit sans voir la cause qui le produit. "Ce qui signifie que l'on donne beaucoup à entendre $-y$ compris des sons anecdotiques lqu'on pense à l'Étude aux chemins de fer de Schaeffer, à la Symphonie pour un homme seul de Schaeffer/Henry, à Hétérozygote de Ferraril - mais rien à regarder. Depuis plus de quarante ans, l'acception s'est considérablement enrichie. 
physique et puissent concentrer leur attention sur le seul contenu de son message. Plus près de nous, au début du siècle, on trouve encore dans le Larousse pour tous en deux volumes: "Acousmate, n.m. Idu grec Akousma, ce qu'on entend). Bruit imaginaire ou dont on ne voit pas les causes, l'auteur." Mais en 1955, l'écrivain et poète Jérôme Peignot, lors des débuts de la musique concrète, reprend l'adjectif acousmatique qui qualifie un son que l'on entend sans en déceler les causes pour désigner "cette distance qui sépare les sons de leur origine" (Peignot, 1955) en occultant derrière l'impassibilité des haut-parleurs tout élément visuel auquel les rattacher. En 1966, Pierre Schaeffer songe un instant à intituler son Traité des objets musicaux, Traité d'acousmatique. Enfin, vers 1974, pour marquer la différence et éviter toute confusion avec les musiques électroacoustiques de scène ou d'instruments transformés londes Martenol, guitares électriques, synthétiseurs, systèmes audionumériques en temps réel...l. François Bayle introduit l'expression musique acousmatique comme spécifique d'une musique qui "se tourne, se développe en studio, se projette en salle, comme le cinéma". Car il est vrai que depuis une vingtaine d'années, sous le vocable électroacoustique, prolifèrent $d^{\prime}$ innombrables productions sonores sans grand rapport les unes avec les autres hormis leur commun recours à l'électricité. Il était donc important d'affirmer par une précision terminologique, des choix esthétiques, une réflexion et un langage.

(Dhomont, 1991a, p. 9.)

Une fois posés ces quelques jalons historiques et principes fondateurs, c'est, bien entendu, vers la problématique ébauchée dans cette dernière phrase qu'il paraît important de se tourner.

La musique électroacoustique est, en effet, si fréquemment réduite par ses détracteurs (mais parfois aussi, hélas! par ses adeptes) à un inexpressif catalogue d'artefacts, de bizarreries acoustiques et d'expérimentations techno-scientifiques qu'on en vient à se demander si le projet musical fait encore partie des intentions de ceux qui la pratiquent.

C'est pourquoi, même si j'en désapprouve la formulation hautaine ef le ton péremptoire, je partage la réprobation lucide de Jean-Baptiste Barrière qui, ici même, page 48, pourfend "la confusion des valeurs typiques des milieux artistiques aux marges de la technologie, mais aussi de l'époque "(3), tout en regrettant que cette condamnation sans appel et sans exception de ses confrères du "ICMC 91 " n'émane pas d'un compositeur dont l'œuvre confirmé soit, lui, inattaquable.
(3) Ce n'est pas la première fois d'ailleurs que cet auteur exprime de telles opinions; il avait déjà fustigé l'ICMC 1986 de la Haye d'une plume vengeresse, dans un texte paru, notamment, dans le Computer Music Journal, où l'on pouvait lire, entre autres douceurs: "(...) il n'est (I'ICMC) que le lieu dévoyé d'une forme moderne de conservatisme, de corporatisme imbécile el borné »; pour le reste, le discours était à peu de chose près identique à celui que nous publions ici. Ce qui m'amène à poser une double question: 1/ Par quelle compulsion masochiste Barrière s'obstine-t-il à fréquenter I'ICMC ? 2) Au-delà de la dénonciation (utile) et du mépris (méprisable), l'auteur ne serait-il pas plus convaincant s'il nous faisait part de ses propres engagements esthétiques autrement qu'à contratio et en les explicitant? 
Pour sa part, l'acousmatique, ou mieux, selon moi: l'«art acousmatique" (Denis Dufour)(4), se préoccupe davantage des fondements ontologiques et des mécanismes perceptifs propres à l'organisation des morphologies qu'à leurs modes de fabrication proprement dits. Sans, bien sûr, négliger l'exploration des outils qui lui sont indispensables, mais qui ne constituent pas la matière de son discours, il privilégie toujours I'« entendre " par rapport au "faire", le perçu par rapport au conçu. On reconnaît ici l'héritage schaefférien.

Ainsi révélées par une écoute privée de toute relation visuelle de cause à effet entre l'être sonore visé et l'agent qui le produit $|5|$ - écoute aveugle, médiatisée, opposée à l'écoute directe, porteuse, elle, de causalités - il était prévisible que les œuvres aient à tenir compte d'une situation aussi insolite et à y définir un territoire spécifique. II ne suffisait pas, en effet, que la musique jaillit des haut-parleurs pour qu'une œuvre fût acousmatique $^{(6)}$; elle ne pouvait se contenter $d u$ seul critère de l'écoute a posteriori et devait répondre à quelques lois internes et principes génératifs: " $(. .$.$) de simple attitude passive de réception, l'acousmatique est$ devenue activité de langage." (Dhomont, 1991b, p. 11.) En effet, les compositeurs sentirent bientôt, au fil de la révélation heuristique, la nécessité d'identifier, de nommer, de formaliser leurs découvertes, les choix qui les guidaient, la nature des matériologies qu'ils polissaient sans cesse ef repolissaient, bref les traits pertinents de cette nouvelle manière si différente de l'ancienne, opposée à elle, même, sous certains aspects.

Nous ne pouvons parcourir ici les étapes successives d'une réflexion en perpétuel mouvement, une telle approche nécessitant des commentaires trop abondants pour trouver leur place dans un exposé aussi lapidaire que celui-ci(7). Me basant sur les conclusions (provisoires) d'un groupe d'étude récemment formé en France ${ }^{(8)}$, je me contenterai de résumer les huit points principaux qui constituent, selon ce groupe de praticiens, les "éléments fondateurs de l'art acousmatique":

$1^{\circ}$ Tout d'abord, bien entendu, le préalable pythagoricien: "Écouter

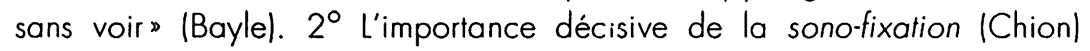
sur un support lanalogique, numérique, peu importe: bande, disquette, disque compact, etc.) - i'y reviendrai. $3^{\circ}$ Un mode de création fondé sur la perception mentale plutôt que strictement auditive lau sens de la psycho-acoustique). $4^{\circ} \mathrm{D}^{\prime}$ où l'on postule l'acousmatique (ses visées, sa conduite d'écoute perceptivel comme discipline phénoménologique. $5^{\circ}$ La problématique causalité/non-causalité et causalité virtuelle des éléments sonores mis en présence ( $d$ 'où peut émerger la narrativité). $6^{\circ}$ Une rhétorique des morphologies et de leurs prégnances. $7^{\circ}$ Une
(4) Michel Chion, de son côté, souhaiterait conserver l'appellation musique concrète, partout reconnue, plutôt que d'égarer les esprits avec un terme nouveau. L'objection souvent opposée à ce choix est la connotation historique associée à concrète qui en fait une tentative appartenant au passé. En ce qui me concerne, je la considère comme bien vivante dans sa forme actuelle, mais je crois que les explications à donner risquent d'être plus déroutantes encore pour l'opinion.

(5) Dont la conséquence ultime et logique sera l' « écoute réduite " de Schaeffer inspirée de la réduction phénoménologique.

(6) À ce titre, toute musique écoutée à partir d'un disque le serait, ainsi que tout son enregistré ou produit électroniquement, tel que par la radio et le téléphone, tant il est vrai que notre époque ne cesse, comme Monsieur Jourdain faisait de la prose, de faire de l'acousmatique sans le savoir. Mais ce qui est acousmatique ici, c'est l'écoute.

(7) On trouvera les développements de ces notions souvent complexes dans les ouvrages qui figurent à la bibliographie.

(8) "ABSO-absolument" /référence non-équivoque à la Symphonie pour un homme seul de Schaeffer-Henryl, mouvement d'Art acousmatique crée en août 1991 à l'occasion des deuxièmes Rencontres de Crest. 
musique du son en tant que matière sonore. $8^{\circ}$ L'espace comme dimension compositionnelle à part entière, de l'élaboration de l'œuvre jusqu'à sa projection en salle $e^{(9)}$.

Je n'ignore pas ce que cette énumération, qui nécessiterait une exégèse approfondie, peut avoir d'abstrait. Elle donne néanmoins, même sous cette forme aphoristique, une assez bonne idée des options incontournables, des mécanismes, du langage et du projet acousmatiques. Au point actuel de la réflexion, ces éléments sont peut être encore incomplets, mais ils semblent rendre compte des principaux cas de figure et susciter à peu près l'adhésion des compositeurs qui se réclament du genre. II s'agit d'une analyse des notions-clés destinée à tracer les lignes de force théoriques d'une praxis partagée, et aussi les frontières de ce qui participe de l'art acousmatique et de ce qui s'en écarte. Parmi plusieurs autres notions importantes qu'il serait trop long de mentionner ici, il faut cependant citer celle, fondamentale, proposée par Bayle, d'«images de son - ou i-sons - en leurs trois espèces: im-son iconique, di-son diagrammatique, mé-son métaphorique» (Bayle, 1991a, p. 131) qui interagit et démultiplie les points 3, 4, 5 et 6 des "éléments fondateurs" présentés plus haut. Notion pénétrante et assez complexe pour faire à elle seule l'objet d'un vaste commentaire dans un ouvrage sous presse (Bayle, 1993). L'«i-son» reprend et transfère à l'image acoustique l'apparent paradoxe visuel de Magritte: "Ceci n'est pas une pipe." II en révèle certains aspects archétypiques dissimulés dans les replis de l'imaginaire.

Devant cet insolent bouleversement du paysage musical, une question (inspirée de Varèse): "Mais est-ce de la musique?" que j'ai moi-même beaucoup posée à une époque, semble gagner aujourd'hui les jeunes acousmates. C'est que l'abandon des syntaxes classiques et des accords classés au profit du règne morphologique ne va pas sans quelques doutes vertigineux. "Peuton donner crédit à une musique des sons les plus généraux, jusqu'aux bruits inclus?" interroge opportunément François Bayle (199lb, p. l) qui suggère, par l'énoncé même, que, pour lui, il s'agit bel et bien de musique. Ce qu'il confirmera d'ailleurs dans un autre texte (1991c, p. 38), renvoyant les adversaires dos à dos en une élégante formule-miroir digne de Salomon, qui accorde aux instrumentaux, lorsqu'ils se préoccupent de sonorité, le son de la musique et aux acousmatiques la musique $d u$ son. Cette formule, que l'on souhaiterait magique, réconciliera-t-elle les "adversaires»? Conservant quelque doute sur l'ouverture des milieux traditionalistes (explicitation ci-dessous), je n'en jurerais pas. Mais il n'est pas interdit de rêver.
(9) D'après l'Absolulettre $n^{\circ} 00$, janvier 1992, D. Dufour, (éd.), Crest, pp. 5-7. 
Si les uns hésitent à prendre leurs distances avec la musique, jugeant cette discrimination trop radicale et maladroite, d'autres, en revanche, fatigués de voir l'acousmatique considérée comme "une pratique dévoyée (...), un sous-produit de la musique instrumentale" (Minjard) ne parle-t-on pas quelque part de *bricolage " ? proposent l'échange du terme musique contre celui d'art et affirment sans équivoque l'autonomie d'un concept nouveau, inhérent au $x x^{e}$ siècle, né de ses découvertes: l'«art acousmatique ». Mais, pour le moment, le consensus reste hésitant parmi les compositeurs et leurs alliés, l'extraction de l'appellation musique s'avérant pour certains aussi douloureuse que celle d'une dent de sagesse. "Se consacrer à l'Art Acousmatique est plein de promesses, mais c'est aussi un renoncement ", avertit François Delalande (1992) dans une récente prise de position dont je reparlerai plus loin.

Bien que cette éventualité "acousmatique versus musique" ne soit pas vraiment nouvelle, elle apparaît comme une position trop anticonformiste pour ne pas provoquer certains remous. Elle va bien au-delà de la simple nuance terminologique et remet en question, non sans quelque provocation, des orientations fondamentales et des habitudes de pensée qui semblaient inviolables. Pour ma part, je dois avover que depuis quelque temps, considérant le pathos - qui semble relever davantage de l'inconscient collectif et du tabou que d'un choix rationnel - que ne manque pas de déchaîner cette question, je n'y consacre plus guère de temps; j'emploie donc indifféremment le mot musique ou art, tout en gardant une préférence marquée pour ce dernier.

En revanche, il m'apparaît essentiel d'assumer sans réserve la notion cardinale de "musique de support versus musique d'interprétation », c'està-dire le principe fondateur de fixation du son. L'acousmatique, descendante directe de la musique concrète, qui "surgit comme l'enfant naturel né d'un couple clandestin: l'art musical el la radiophonie" (Bayle, 1991 b, p. 31, n'est concevable qu'à partir du moment où les techniques d'enregistrement permettent de conserver une trace, une image fidèle des phénomènes sonores, manifestations éphémères, volatiles qui, jusqu'à l'avènement de la captation du son, à peine apparus disparaissaient à jamais. Car si la technologie paroxystique peut donner lieu, nous l'avons vu, à des aberrations, il ne faut pas oublier que sans elle plusieurs des arts majeurs de notre siècle n'existeraient pas. Je pense, bien entendu, au cinéma, mais aussi aux nombreux arts médiatiques qui ont métamorphosé l'environnement sensible de notre époque. L'acousmatique est de ceux-ci. Qu'on me permette une nouvelle auto-citation: 
La découverte qui permet au phénomène audible d'échapper à la disparition ne pouvait rester neutre. Pense-ton qu'elle soit sans effet sur notre perception auditive du monde? N'est-elle pas assez bouleversante pour provoquer une mutation ou, au moins, de considérables changements dans l'art des sons?

(Dhomont, 1991b, p. 12.)

Le détournement génial, c'est d'avoir fait d'un moyen de reproduction sonore un outil de production artistique. À la différence de la musique instrumentale qui opère une lecture herméneutique de notations symboliques, l'acousmatique ne donne pas à entendre quelque chose qui ressemble, même de très près, à ce qu'a voulu le compositeur, c'est ce qu'il a voulu. Cette réplique du son, beaucoup plus précise que n'importe quelle notation écrite (10), restitue en effet avec une fidélité incomparable les moindres intentions de l'auteur. De plus, en ne dirigeant pas l'écoute vers des paramètres que met en évidence une représentation graphique, elle autorise tous les parcours de l'imaginaire en fonction des visées différentes qui seront choisies. Ce que confirme Lelio Camilleri à propos de l'analyse des œuvres électroacoustiques:

(...) I'unique texte analysable est le texte sonore. Cette caractéristique représente, selon moi, un point de force et non de faiblesse de ce genre musical. Se concentrer seulement sur la matière sonore sans avoir aucune référence écrite évite le risque de réaliser des analyses basées seulement sur un aspect (celui des relations de hauteurs, par exemple) qui souvent font abstraction de ce qui s'entend réellement.

(Camilleri, 1991, p. 45.)

Ces œuvres répondent donc à des intentions d'écoute nouvelles; elles ne se préoccupent plus de combinatoires et de permutations d'unités discrètes mais explorent le champ heuristique d'une sémiologie d'êtres sonores "spectro-morphologiques" (Smalley) si complexes que malgré leur gel définitif, ils pourront être perçus de cent façons différentes. L'acousmatique, c'est l'art des représentations mentales - figuratives ou abstraites suscitées par le son.
(10) Relevons au passage la contradiction grossière qu'il y a à prétendre, comme le font certains de ses adversaires, que la musique acousmatique est imprécise (parce que non écrite sur une partition) et, en même temps, qu'elle est figée Iparce que reproductible dans ses plus infimes détails grâce au support). 


\section{et défense de l'acousmatique (où l'on appelle un chat, un chat)}

Mais la polémique n'est pas seulement le plaisir de faire des mots; c'est avant tout une tentative pour imposer des idées justes.

(Boulez, 1986.)

Pourquoi, dans ces conditions, tant de réticences? Que reproche-t-on à l'acousmatique, art ou musique? Oublions les sarcasmes éculés sur la musique concrète mais prêtons l'oreille aux arguments de la "doxa " et du "bon sens" musical. L'un des principaux griefs concerne l'absence d'interprète et de spectaculaire; et il est vrai qu'aucun trapéziste ne vient plus faire la preuve de sa virtuosité au risque de s'écraser sous nos yeux. II faut admettre aussi que, du point de vue strictement visuel, l'intérêt d'un "orchestre de haut-parleurs" reste mince. Quant à l'obscurité, qui favorise indiscutablement la perception de la musique et des images mentales, elle surprend et parfois inquiète. Cependant, personne ne s'avise de condamner l'art plastique sous prétexte qu'il n'offre pas, dans sa représentation, d'adjuvant sonore ou d'exhibition en direct; l'art des sons et des durées serait-il moins autonome que celui des surfaces et des volumes? II s'agif donc bien d'un comportement lié à la force de l'habitude et à la convention sur lequel viennent en général se briser les arguments rationnels. Mais peuton considérer l'obstination comme un critère pertinent?

Une objection corollaire de la précédente et particulièrement mal fondée consiste à décréter qu'ainsi fixée pour ne plus changer, la musique devient aussi inerte qu'un papillon épinglé sur son bouchon. "Une bande est une chose morte parce qu'on ne peut rien ajouter au code purement quantitatif qu'elle contient», tranche Boulez (1992, p. 20) qui, depuis son fameux "Schœnberg est mort", a quelque propension à occire ce qui ne lui sert plus à rien. On a pu lire plus haut ce que vaut cette affirmation pour peu qu'on la considère avec sérieux.

Autres difficultés pour une oreille traditionnelle, le rôle mineur dévolu ici aux habituels jeux de hauteurs (disparition du "mélodique") ou à la pulsation rythmique basée sur les divisions métriques et la répétition. Ces valeurs du musical (peuton prétendre qu'elles sont des universaux?), tenues jusqu'alors pour incontournables, cèdent la place aux caractères du sonore, au travail sur les morphologies, à la prégnance du geste, à l'exploration des structures internes du son ef à la rémanence de leur 
gestalt dans notre psyché. C'est en cela qu'on peut estimer qu'il s'agit d'une rupture radicale sans précédent (et donc peutêtre d'un art nouveau), et non, comme le soutient Delalande (1992), d'un changement, certes considérable, comparable à celui de l'Ars Nova (l'I), mais qui n'échappe pas au processus évolutif de l'histoire de la musique.

Tels sont les habituels reproches (plus quelques autres) auxquels nous ne cessons, depuis des années, d'apporter des réponses. Mais la force d'inertie et la mauvaise foi ont la vie dure et ne peuvent manquer, à la longue, de déclencher une attitude polémique. Surtout lorsque aux divergences esthétiques s'ajoute une politique d'exclusion, insidieuse, accompagnée de critiques sur le comportement même des acousmates. Qu'ontils donc à être toujours sur la brèche, à se croire menacés, à crier à la persécution? Quel mauvais goût, en effet, et surtout quelle déplorable stratégie! Une attitude plus cool, positive, inspirée des méthodes du marketing, serait, certes, plus porteuse! Et pourquoi marteler sans cesse les mêmes arguments? "Je dis toujours la même chose, répond à Don Juan le Sganarelle de Molière, parce que c'est toujours la même chose."

Demander à l'acousmatique de rentrer sagement dans le rang et de faire confiance à l'histoire, comme le suggère François Delalande dans sa "Harangue (1992), ou à l'angélisme du discours dominant revient à peu près à conseiller à des grévistes de mettre en sourdine leurs revendications sous prétexte qu'elles desservent leur image de marque et que les choses s'arrangeront d'elles-mêmes: "Pourquoi ces gens-là crient-ils si fort, après tout, et de quoi se plaignentils? S'ils avaient de bonnes raisons, cela se saurait. "Ainsi s'articule toujours le sermon officiel. Qui s'est un peu frotté aux luttes sociales, politiques ou écologiques sait bien que ce genre de déni constitue l'argument qui leur est invariablement opposé dans un premier temps: on commence par refuser d'admettre qu'il y ait problème ; le râleur doit toujours avoir tort de râler, c'est en tout cas ce que s'efforce de prouver celui contre qui râle le râleur. Ces mécanismes sont si connus que j'ai scrupule à les rappeler. Nous savons très bien, même s'il est de bon ton de feindre pudiquement le contraire, que la plupart des rapports humains sont des rapports de forces; nous pourrions tous citer cent cas où la justice la plus élémentaire $n$ 'aurait jamais été rendue sans les efforts disproportionnés et obsessionnels de ceux qui ont exigé qu'elle le fût.

On peut le regretter mais, de nos jours, la demi-teinte passe inaperçue, elle est laminée par le tumulte médiatique. "Pour faire entendre sa voix, surtout quand on est dans une situation minoritaire, il faut parler fort. $\dot{A}$ certains moments une idée passe mieux si elle est énoncée de façon plus acérée. (...) Pourquoi voudriez-vous que l'on encaisse les coups sans les
(11) Comparaison que je ne saurais rejeter puisque je l'ai moi-même suggérée dans une œuvre, Novars. 
rendre? " Voilà un discours batailleur et un peu paranoïaque qui ne peut qu'isoler son auteur, ricaneront les bien-pensants. II s'agit, en l'occurrence, d'une opinion de Pierre Boulez (Lesure, 1986, p. 126), encore lui, et quelles que soient les contestations (même justifiées) qui s'élèvent maintenant à son sujet, c'est bien à ses excès polémiques que les faits ont donné raison, légitimant a posteriori son intransigeance pour la cause qu'il défendait. Aurait-il dû suivre le troupeau et faire ce que faisait tout le monde? Un combat n'est jamais compris que lorsqu'il est gagné.

Or voici les visionnaires d'hier, aujourd'hui frappés de cécité, qui refusent d'accorder à une pensée nouvelle, différente de la leur, l'acousmatique, la place qui lui revient ou affectent même d'ignorer son existence. Éternel retour ? Troublante propension des minoritaires sortis de leur ghetto à retomber dans les errements qu'ils ont combattus, englués à leur tour dans les contradictions du pouvoir et d'un nouvel académisme!

J'exagère? Qu'on en juge. Peuton faire semblant d'ignorer la méfiance, voire la répugnance fréquemment rencontrées dans les milieux traditionnels de la musique (toutes professions confondues: compositeurs, instrumentistes, professeurs, musicologues, organisateurs, journalistes, producteurs de radio/télévision, etc.) à l'égard de l'électroacoustique? Cela est frappant dans la plupart des manifestations de musique contemporaine où les œuvres appartenant à ce genre - notamment acousmatiques - sont, avec une persistance qui ne doit rien au hasard, programmées dans les conditions les plus dissuasives (heures impraticables, lieux bruyants, conditions techniques improvisées, émissions de radio à audience minimale, etc.). Dans tout festival, lorsque plusieurs concerts ont lieu dans la journée, où pense-t-on que les œuvres pour bandes seront placées? Toujours au moment le moins accessible au public et annoncées par une promotion minimum(12). Cette façon militante de minorer l'acousmatique, d'insinuer, en arguant de sa marginalité, qu'elle ne saurait occuper le même espace que la "musique-musique", contribue à la marginaliser davantage encore et contraint ses représentants à se soumettre ou à livrer combat. J'ai le mauvais esprit de penser que ce ne sont pas là pratiques innocentes et j'attends qu'on me prouve le contraire. (En ce domaine, le Québec semble néanmoins, il faut le souligner, de façon générale plus enclin que d'autres à donner à l'acousmatique la place qui lui revient.)

On peut ajouter que les concerts électroacoustiques, a fortiori s'ils ne mettent pas en vedette des instrumentistes, ne sont pratiquement jamais couverts par la presse écrite, et lorsqu'ils le sont, c'est en général avec une grande incompétence que ne parvient pas à dissimuler le ton péremp-
(12) Près de nous, on peut citer l'exemple du festival Montréal Musiques Actuelles 1991 (cf. Circuit, vol. $\left.1, n^{\circ} 2\right)$ ou se demander combien d'œuvres acousmatiques ont été inscrites, en vingt-cing ans d'exercice, aux programmes de la Société de musique contemporaine du Québec. Mais ce ne sont que deux cas parmi une quantité d'autres Icomme l'évacuation pure et simple de la musique sur bande aux Journées mondiales de la musique, Varsovie 92 et Mexico 93) qu'il serait fastidieux d'énumérer. 
toire généralement adopté. Car bien rares sont les critiques qui ont accepté que quelque chose, depuis bientôt cinquante ans, ait changé au royaume de la "musique de notes"; leurs jugements, inspirés de modèles dépassés et de grilles d'analyse non pertinentes, sont en général empreints d'un prétentieux conformisme. Plus rares encore sont ceux qui prennent la peine de recycler leurs connaissances. "Des noms! des noms!" hurle la foule. Eh bien, je ne vois guère à Montréal que Carol Bergeron, du Devoir, qui, précisément, se soit fait un devoir de s'informer avec honnêteté et de couvrir régulièrement les manifestations d'un genre qui a quand même quelque peu secoué le train-train musical depuis la moitié du siècle. À Paris, après la disparition de Maurice Fleuret et le départ à la retraite de Jacques Lonchampt, reste-t-il quelqu'un(13) ? Les absents d'ici et de là-bas se reconnaîtront (14).

Que nous proposent, d'autre part, les stratèges de service? De calmer notre émoi et de remettre notre sort entre les mains de gens qui, avec une ardente mauvaise volonté, depuis plus de quarante ans, n'ont pas manqué une occasion de dénigrer nos idées, nos œuvres, nos recherches et qui, ne parvenant pas à les évacuer et encore moins à prendre le pas sur elles, ont entrepris de s'en attribuer les mérites sans, bien entendu, en reconnaître la paternité à leurs auteurs véritables lie ne parle pas ici d'électroacoustique at large mais bien d'un art des sons fixés que l'on voudrait confondre avec le "reste"). Aussi, lorsque François Delalande, ce vieux compagnon de route de la "concrète" - soudainement charmé par le chant de quelles sirènes? - s'efforce d'apaiser notre courroux: "On peut prévoir que la problématique musicale de la création instrumentale, même si celle-ci continue à tenir le haut du pavé (c'est moi qui souligne), sera de plus en plus dérivée de la problématique de la musique de support", i'éprouve quelque perplexité devant les motifs de satisfaction que devrait provoquer une telle éventualité. Pense-ton que l'honneur d'être spoliés par l'institution musicale en place, qui cautionne ainsi le bien-fondé de nos idées en se les appropriant, suscite chez nous un sentiment de gratitude tel que nous soyons disposés à lui servir de nègres? Cette carotte illusoire devrait-elle nous faire oublier le bâton qui nous chasse des lieux de la musique? Et, puisqu'il est question pour notre ami de stratégie, estime-t-il vraiment que la complaisance soit une stratégie payante? Peut-il ne pas avoir remarqué, par exemple, que l'ouvrage dont il s'inspirel(5) pour prédire l'avènement de nos thèses ne donne la parole à aucun de nos chercheurs, de nos compositeurs, de nos théoriciens, pas même à lui, qui pourtant montre patte blanche? «Comme c'est étrange, comme c'est bizarre et quelle coïncidence!" Est-ce crédible de penser qu'il ignore avec quelle persévérance, depuis plus de quarante ans, l' «establishment"
(13) Si l'on excepte Michel Chion qui assura jusqu'à l'an dernier une chronique engagée dans le Monde de la musique mais qui, comme on dit, a fait partie de la maison». Je ne puis me prononcer sur la situation internationale. (14) Mais il est réconfortant et peut-être révélateur d'un changement d'attitude chez la génération montante des compositeurs de lire dans Circuit (vol. III, $n^{\circ}$ 1) les réponses de Gonneville, Lalonde et Lesage aux habituels anathèmes lancés par Boulez contre les musiques pour bande. Saluons ces prises de position rafraîchissantes et rendons hommage à l'équipe de cette même revue qui nous donne aujourd'hui la parole.

(15) Jean-Baptiste Barrière (1991) (éd.), Le Timbre, métaphore pour la composition, Paris, Christian Bourgois éditeur. 
musical n'a pas manqué une occasion de ricaner devant nos idées, nos œuvres, nos recherches? Voyons, cher François, est-ce que je rêve et parlez-vous sérieusement? La spéculation historique vers le futur suppose quand même une appréciation juste du présent.

Et qu'on ne vienne pas me dire que nous avons désormais pignon sur rue, que notre pratique est partout reconnue, à commencer par des instituts en renom. C'est précisément l'une des manœuvres dilatoires les plus courantes et les plus hypocrites que de favoriser l'amalgame avec l'ensemble de la production électroacoustique pour refuser à l'acousmatique son statut de "société distincte". On peut de cette façon la réduire au silence tout en faisant état du crédit grandissant accordé à l'ensemble des nouvelles techniques musicales. Mais, dans la plupart des cas, il ne s'agit alors que d'un prolongement technologique de la pensée, du langage et des besoins instrumentaux traditionnels. Rien de plus, en général, pour certains "musiciens d'écriture", ceux qui gèrent ou fréquentent ces instituts célèbres, qu'une extension électronique de la lutherie qu'ils pratiquent, un simple mais indispensable ravalement de la façade instrumentale ${ }^{(16)}$.

Devrions-nous alors, à notre tour, ignorer ceux qui nous ignorent? Assurément pas. Ce serait répondre à l'intolérance par la bêtise, remplacer un sectarisme par un autre. La reconnaissance réciproque des genres constitue désormais la seule perspective d'avenir réaliste, ce que le Québec semble avoir compris. Guérirons-nous enfin des exclusives et des intégrismes?

En guise d'avertissement, cette hypothèse risquée: peut-être aussi les patriciens de la musique, sentant les hordes se presser aux portes de l'empire, se raidissentils sous la menace. Rome, accrochée à ses privilèges, ne sut pas davantage partager avec ses barbares la force vive qu'elle leur arrachait. Son déclin fut inexorable. Les censeurs d'aujourd'hui seraient bien inspirés de s'en souvenir.
(16) Cf. I'article de P. Boulez « Zukunftsmusik? Avenir de la musique? " et le mien, "Repenser la musique aujourd'hui», Circuit, vol. III, $\mathrm{n}^{\circ}$ l.

Absolulettre, n० 00 (janvier 1992), compte rendu des deuxièmes Rencontres d'art acousmatique de Crest, France.

BAYLE, F. (1985): «la musique acousmatique ou l'art des sons projetés», Encyclopæedia universalis, Symposium, pp. $211-218$. 
BAYLE, F. (1991 a), "Mi-lieu', in F. Dhomont, (éd.), L'Espace du son II, Ohain (Belgiquel, éditions Musiques et Recherches, pp. 131-135.

BAYLE, F. (199/b), Rimage ou la Transgression acousmatique, texte inédit devant paraître dans la revue italienne Musica realtà, Milan.

BAYLE, F. (199/c), "Mon (ton, son) acousmatique?", in A. Vande Gorne (éd.), Vous avez dit acousmatique?, Ohain (Belgique), éditions Musiques ef Recherches, pp. 37-38.

BAYLE, F. (1993), Musique acousmatique, propositions... positions, Paris, INA-GRM, Buchet-Chastel éditeurs.

BOULEZ, P. (1992), "Zukunftsmusik, avenir de la musique? \&, Circuit, vol. III, n० 1, pp. 7-21.

CAMILLERI, L. (1991), :La musica elettroacoustica, I'analisi e i processi d'ascolto". Sonus, vol. 3, n०2, Materiali per la musica contemporanea, pp. 44-56.

CHION, M. (1991), L'Art des sons fixés ou la Musique concrètement, Fonlaine, France, Éditions Métamkine/Nota-Bene/Sono-Concept.

Le Concert, pourquoi? Comment? (1977), M. Chion (ed.), GRM, Institut national de l'audiovisuel éditeur, Paris.

DELALANDE, F. (1992), "Fautil abandonner le rerrain ₹ Harangue aux militants d'un art acousmatique», in D. Dufour (éd.), Absolulettre no 1, Rencontres d'art acousmatique, Crest.

DHOMONT, F. (1991 a), "Acousmatique, qu'est-ce à dire", in J.-F. Denis (éd.), MouvancesMétaphores (livret du double CD Dhomont), Montréal, empreintes DIGITALes, pp. 9-10.

DHOMONT, F. (1991b), "L'acousmatique: une projection vers le futur", in A. Vande Gorne (éd.), Vous avez dit acousmatique?, Ohain (Belgique), éditions Musiques et Recherches, pp. 11-12.

LESURE, F. (1986), “La vérité violente", in C. Samuel (éd.), Éclats/Boulez, Paris, Éditions du Centre Pompidou, pp. 124-127.

PEIGNOT, J. (1955), "D’où vient le mot acousmatique", Musique animée, quinze émissions radiophoniques, chaîne nationale, France.

- Recherche musicale au GRM *, (1986), M. Chion ef F. Delalande (éd.), La revue musicale, quadruple numéro 394, 395, 396, 397, Paris, Éditions Richard Masse.

Réel/Virtuel, cinq questions d'acousmatique (1992), débats du Cycle acousmatique SON-MU, INA-GRM, Paris.

Vous avez dit acousmatique? (1991), A. Vande Gorne (éd.), Ohain, Belgique, éditions Musiques et Recherches. 